\title{
MEMÓRIA DE TRABALHO E CONSCIÊNCIA FONOLÓGICA NO DESVIO FONOLÓGICO
}

\author{
WORKING MEMORY AND PHONOLOGICAL AWARENESS IN THE \\ PHONOLOGICAL DEVIATION
}

\author{
Michele Gindri Vieira*
}

\begin{abstract}
Resumo: Este estudo investiga o desempenho de crianças com desvio fonológico nas habilidades em memória de trabalho e em consciência fonológica, as quais fazem parte do processamento fonológico. Além disso, verifica se tais habilidades estão relacionadas entre si, com a idade e com a severidade desse desvio. A amostra foi formada por 28 crianças com desvio fonológico, com idades entre 4 anos e 6 anos e 7 meses, não alfabetizadas, com hipótese de escrita pré-silábica, as quais foram divididas em dois grupos: um, com 21 crianças com desvio fonológico mais severo; outro, com 7 crianças com desvio mais leve. Avaliações fonoaudiológicas e audiológica foram realizadas para obter-se diagnóstico do desvio fonológico, juntamente com avaliações fonológicas que determinam o sistema fonológico e classificam a severidade do desvio por meio de medida qualitativa. 0 desempenho das crianças, na avaliação da memória de trabalho, foi verificado através da tarefa de repetição de sequências de dígitos - para avaliar o executivo central e a memória fonológica - e por meio da tarefa de repetição de não palavras - para avaliar especificamente a memória fonológica-, segundo a proposta de Baddeley e Hitch (1974 apud GATHERCOL; BADDELEY, 1993), revisada por Baddeley (1986). Para analisar o desempenho na avaliação de consciência fonológica, incluíram-se tarefas de consciência silábica e de fonêmica. Nos resultados, verificou-se: 1) as crianças que participaram da amostra apresentaram desempenho inferior em memória fonológica e em consciência fonológica quando comparadas a crianças com desenvolvimento fonológico normal que participaram de outras pesquisas brasileiras com metodologias de avaliação semelhantes; 2) houve correlação significativa entre a tarefa de repetição de não palavras e a de consciência de sílabas; 3 ) houve correspondência significativa entre idade e tarefa de repetição de não palavras e fraca relação entre idade, repetição de dígitos e consciência fonológica. Na comparação entre os grupos, verificou-se que as crianças com desvio fonológico mais severo apresentaram desempenhos inferiores em todas as tarefas. Essa diferença é significativa aos escores totais de consciência fonológica e à consciência fonêmica. Concluiu-se que crianças com desvio fonológico em idade pré-escolar, quando consideradas como grupo e não individualmente, apresentaram pior desempenho do que grupos de crianças com desenvolvimento fonológico normal em tarefas de consciência fonológica e em memória fonológica. Não obstante, podese inferir que tais habilidades estão significativamente correlacionadas, posto que crianças com desvio fonológico mais severo apresentam pior desempenho do que crianças com menos alterações na fala, com diferença estatisticamente significativa nas tarefas de consciência fonológica.
\end{abstract}

Palavras-chave: Memória de trabalho; Consciência fonológica; Desvio fonológico.

\footnotetext{
${ }^{*}$ Fonoaudióloga, com Especialização em Fonoaudiologia (UFSM), Mestre em Distúrbios da Comunicação Humana pelo Programa de Pós-graduação em Distúrbios da Comunicação Humana (UFSM) e Doutoranda no Programa de Pós-graduação em Linguística (UFSC).
} 


\begin{abstract}
This study investigated the performance of children with phonological disorders in the working memory skills and in the phonological awareness skills, which are parts of the phonological processing and, whether such skills are related to each other, to the chronological age and to the degree of the phonological deviation. The sample was composed of 28 children with phonological deviation, ranging from 4 years old to 6 years and 7 months old, illiterate, with a hypothesis of pre-syllabic writing and, it was divided into two groups as follows: one with 21 children with a more severe phonological deviation and the other with 7 children with a softer phonological deviation. Speech-language and hearing evaluations were undertaken in order to diagnose the phonological deviation along as phonological assessments to determine the phonological system and to rate the degree of the speech impairment through a qualitative analysis. The children's performance in the working memory assessments was checked through the repetition of a sequence of four digits - to assess the central executive and the phonological memory and, the repetition of non-words task - to assess specifically the phonological memory, according to Baddeley and Hitch's proposal (1974 apud GATHERCOLE; BADDELEY, 1993), revised by Baddeley (1986). The performance in the phonological awareness evaluation was also checked, including the syllabic and phonemic awareness. The results are as follows: 1) the sample has presented a lower performance in the phonological memory and phonological awareness when compared to the performance of children with normal phonological development as seen in further research conducted in Brazil by making use of similar assessment methods; 2) there has been a significant correlation between the repetition of non-words task and the syllable awareness; 3) there was a statistical correlation between age and the repetition of nonwords task and, a weak correlation between age and the digit repetitions and, between age and phonological awareness. Comparing the groups was found that the children with a more severe phonological deviation have performed lower in all the tasks when compared to the group of softer deviations and, such difference was significant in the total scores of the phonological and in the phonemic awareness. It concluded that the preschoolers with phonological deviation, when considered as a group and not individually, have presented worse performance in tasks of phonological awareness and phonological memory than groups of children with normal phonological development and, that such skills are correlated in a significant way. Children with more severe phonological deviation have presented worse performance than children with less speech impairments, with a statistical difference in the phonological awareness tasks.
\end{abstract}

Keywords: Working memory; Phonological awareness; Phonological deviation.

\title{
Introdução
}

A interface linguística e a fonoaudiologia têm, como área de interesse comum, a fonologia, sua aquisição e seu desenvolvimento, em seus aspectos típicos e atípicos. As pesquisas linguísticas sobre aquisição e desenvolvimento fonológico auxiliam a fonoaudiologia clínica a desenvolver tratamento mais eficaz do desvio fonológico; em contrapartida, a atuação fonoaudiológica voltada à pesquisa auxilia a linguística em sua teorização.

A dificuldade das crianças com desvio fonológico parece centrar-se nas habilidades fonológicas e no processamento dessas informações, como memória de trabalho e de consciência fonológica. Visto que as habilidades em memória de trabalho e em consciência fonológica fazem parte do processamento fonológico e são funções 
cognitivas, busca-se esclarecer a relação entre ambas as habilidades nas crianças que apresentam desvio fonológico.

Memória fonológica e consciência fonológica compartilham componentes de processamento fonológico. Supõe-se que a memória fonológica seja fator importante e necessário para a execução de tarefas que avaliam a consciência da estrutura sonora das palavras e, por isso, requer uma análise mais detalhada da natureza segmentar e da fonêmica da linguagem.

Entender a relação entre a memória fonológica e a consciência fonológica, em crianças com desvio fonológico em idade pré-escolar, auxiliaria a detecção precoce de déficits dessas habilidades, por meio de avaliações aplicadas antes da alfabetização e por intermédio de programas de intervenção na consciência fonológica e na memória, e para integrar à terapia fonológica.

Assim, seria possível amenizar e até mesmo prevenir problemas de alfabetização. Desse modo, proporcionar-se-ia melhor condição de aprendizagem às crianças que têm dificuldade nas habilidades em consciência fonológica e/ou em memória de trabalho associada ao desvio fonológico, tanto no contexto clínico quanto no educacional.

Este estudo tem por objetivo tanto investigar o desempenho de crianças com desvio fonológico, não alfabetizadas, com idades entre 4 anos e 6 anos e 7 meses, em habilidades cognitivas em memória de trabalho e em consciência fonológica, quanto verificar de que modo essas habilidades estão relacionadas entre si, com a idade cronológica e com a severidade do desvio fonológico.

Para auxiliar a compreensão do leitor, serão definidos primeiramente os termos utilizados no decorrer deste artigo, como desvio fonológico e processamento fonológico, que abarcam o conceito de memória de trabalho e de consciência fonológica. A seguir, serão apresentados a metodologia da pesquisa, os resultados obtidos e a discussão sobre o assunto, para, posteriormente, expor-se as conclusões resultantes do trabalho.

\section{Desvio fonológico}

A mudança de paradigma sobre o distúrbio de fala, o qual se considerava decorrente apenas do aspecto motor, ocorreu a partir da década de 70. Foi com Ingram (1976) que se percebeu que esse distúrbio é proveniente da dificuldade que a criança 
tem em se adequar ao sistema fonológico padrão da comunidade linguística na qual está inserida.

O mesmo ponto de vista é defendido por Grunwell (1981), que se refere ao desvio fonológico como desordem linguística que se manifesta pelo uso de padrões incorretos no meio falado da linguagem. 0 desvio fonológico caracteriza-se como uma dificuldade específica para o aprendizado da fala na ausência de fatores etiológicos conhecidos e detectáveis, como, por exemplo, dificuldade geral de aprendizagem, déficit intelectual, desordens neuromotoras, distúrbios psiquiátricos ou fatores ambientais.

Acosta et al. (2003) afirmam que o processo de aquisição fonológica começa desde o nascimento e continua de forma progressiva até a idade de quatro anos, aproximadamente, na qual a maioria dos sons surgem discriminados em palavras simples. Esse desenvolvimento completa-se quando ocorre o domínio de determinadas estruturas silábicas e de fonemas mais complexos, por volta dos seis ou sete anos, no caso do espanhol. Segundo Oliveira et al. (2004), no caso do português brasileiro, por volta dos 5 anos o onset complexo, estrutura mais complexa, é adquirido.

Segundo Lamprecht et al. (2004), o desenvolvimento fonológico é um processo que ocorre gradualmente, de forma não linear, com variações individuais para a maioria das crianças até a idade aproximada de cinco anos. 0 resultado desse desenvolvimento é o estabelecimento de um sistema fonológico condizente com o do adulto, fato que não ocorre com crianças com desvio fonológico, pois formulam um sistema inadequado em relação à fonologia da língua do seu ambiente.

Não há resultados conclusivos a respeito da etiologia do desvio fonológico, mas, segundo Wertzner (2009), alguns fatores têm sido associados ao gênero, à otite, à alteração de vias aéreas superiores e ao histórico familiar. 0 desvio fonológico pode ser classificado de acordo com sua gravidade. Uma proposta quantitativa muito citada na literatura é a de Shriberg e Kwiatkowski (1982), revista por Shriberg et al. (1997), que utiliza a medida de Porcentagem de Consoantes Corretas (PCC) para classificar o desvio fonológico em leve (PCC de 85 a 100\%), levemente moderado (PCC entre 65 e 85\%), moderadamente grave (PCC entre 50 e 65\%) e grave (PCC abaixo de 50\%).

Pode-se também classificar o desvio qualitativamente. Keske-Soares (2001) estabeleceu um critério de caracterização por tipologia - que será utilizado neste trabalho -,com base em características encontradas nos sistemas fonológicos das 
crianças. Mota et al. (2008) encontraram correspondência, estatisticamente significativa, entre a medida quantitativa (PCC) e a medida qualitativa de Keske-Soares (2001) para o desvio fonológico.

A classificação de Keske-Soares (2001) divide os desvios fonológicos em quatro grupos: desvios fonológicos com características incomuns, desvios fonológicos com características iniciais, desvios fonológicos com características atrasadas e desvios fonológicos com características fonéticas adicionais.

No grupo com características incomuns, estão as crianças que apresentam sistema fonológico bastante defasado. Essas crianças realizam processos incomuns (fricatização, glotalização, apagamento de fricativa/plosiva) e têm preferência sistemática por um único som. Observa-se severa ininteligibilidade da fala e, em geral, somente os familiares identificam a fala.

As crianças do grupo com características iniciais de desvio apresentam sistema fonológico com características típicas daquelas em desenvolvimento incipiente. Os processos iniciais da aquisição fonológica persistem além da idade esperada para sua supressão, como plosivização, anteriorização de plosiva e dessonorização. A ininteligibilidade da fala apresenta-se semelhante a de uma criança com idade bem inferior.

No grupo com características atrasadas, o sistema fonológico da criança apresenta simples atraso, com alterações evidenciadas no estágio final da aquisição fonológica, que envolvem as fricativas palatais, as líquidas e as noções de estrutura silábica. Nesse caso, ocorrem processos comuns da fala infantil (anteriorização de fricativa, apagamento de líquida não lateral e redução de encontro consonantal).

As crianças do grupo com características fonéticas adicionais apresentam fatores fonéticos que interferem no desenvolvimento e adequação do sistema fonológico, tais como: freio lingual curto, otites de repetição, amígdalas hipertróficas etc. Tais características podem estar associadas a qualquer um dos três grupos citados.

\section{Processamento fonológico}

Segundo Torgensen, Wagner e Rashotte (1994), o processamento fonológico se refere às operações mentais do processamento de informações baseadas na estrutura 
sonora ou fonológica da linguagem oral. Os autores mencionam que três tipos de habilidades em processamento fonológico estão relacionados com as diferenças individuais existentes na velocidade do aprendizado da leitura e da escrita: velocidade de acesso à informação fonológica (acesso ao léxico mental), memória fonológica e consciência fonológica.

A memória fonológica refere-se ao processamento ativo e ao armazenamento transitório das informações fonológicas (EYSENCK; KEANE, 1994). Reflete também habilidades de representar mentalmente características fonológicas da linguagem (TORGENSEN; WAGENR; RASHOTTE, 1994).

\subsection{Memória de trabalho}

Gathercole e Baddeley (1993) afirmam que, de acordo com o modelo de memória de trabalho, a memória imediata para material verbal é mediada pelo executivo central e pelo circuito fonológico.

O modelo de Baddeley e Hitch (1974 apud GATHERCOLE; BADDELEY, 1993), revisado por Baddeley (1986; 2003), descreve a memória de trabalho como um sistema de memória de curto prazo que está envolvido no processamento temporário e na estocagem da informação. Ademais, desempenha papel importante, posto que serve de subsídio para diversas atividades cognitivas diárias, como o raciocínio, a compreensão da linguagem e o aprendizado em longo prazo (EYSENCK; KEANE, 1994; BADDELEY, 1998). É constituído por três principais componentes: o executivo central e seus dois sistemas subordinados, o circuito fonológico e o registro visuo-espacial.

O executivo central cumpre funções como regular o fluxo dentro da memória de trabalho, recuperar informações de outros sistemas de memória - como o de longo prazo -, processar e armazenar informações (GATHERCOLE; BADDELEY, 1993). A fim de integrar os vários níveis, o executivo central transfere itens para e da memória de curto prazo e integra as informações que chegam dos sentidos e da memória de longo prazo (STERNBERG, 2000). 0 registro visuo-espacial processa e armazena informações visuais, espaciais e o material verbal que será codificado em forma de imagem. 
Neste trabalho, o termo memória fonológica - também denominada memória de trabalho fonológica ou memória de curto prazo verbal - será utilizado para designar o componente da memória de trabalho usado para processar o material verbal.

As tarefas mais utilizadas para investigá-la incluem medidas de digitspan e de repetição de não palavras. 0 digitspan é o número máximo de dígitos falados que alguém pode relembrar e repetir imediatamente na mesma ordem (GATHERCOLE; BADDELEY, 1993) - neste trabalho, chamado de tarefa de repetição de dígitos. Tal tarefa mostra-se eficiente para avaliação da memória de trabalho e tem sido largamente utilizada no Brasil (IZQUIERDO, 2002). Neste trabalho, utilizou-se da tarefa de repetição de não palavras, a qual também é frequentemente utilizada, em pesquisas estrangeiras e brasileiras, para avaliação da memória fonológica.

\subsection{Consciência fonológica}

A consciência fonológica faz parte da consciência metalinguística e é considerada a habilidade em identificar, refletir e manipular, de forma deliberada, tanto os componentes fonológicos das unidades linguísticas (GOMBERT, 1992; TUNMER; HARRIMAN; NESDALE, 1988), como a estrutura de um enunciado distintamente de seu significado (STACKHOUSE, 1997).

Capellini e Ciasca (2000) afirmam que a consciência fonológica permite refletir sobre as características estruturais da fala, incluindo a consciência de que as palavras são constituídas por uma sequência de sons que podem ser manipulados. Moojen et al. (2003) acrescentam que a consciência fonológica abrange não só a capacidade de refletir (constatar e comparar), mas também a de operar com fonemas, sílabas, rimas e aliterações (contar, segmentar, unir, adicionar, suprimir, substituir e transpor).

Para Stackhouse (1997), a consciência fonológica desenvolve-se ao longo de um contínuo de níveis de consciência, da implícita para a explícita, como resultado de experiências auditivas e articulatórias de leitura e de escrita, com a seguinte hierarquia, que é relacionada ao aumento dessas experiências: segmentação silábica, rima, segmentação sonora, manipulação sonora e segmentação de grupo consonantal.

Conforme Cielo (2001), é provável que o desenvolvimento desse contínuo evolutivo seja resultado do maior número de sinapses neuronais que se constituem 
mediante estímulos que aumentam a experiência da criança, além, claro, do funcionamento paralelo e distribuído do cérebro. A emergência das habilidades em consciência linguística é resultado da interação entre fatores maturacionais, linguísticos e cognitivos de aprendizagem do código escrito e de interação com o meio ambiente.

Para Avila (2004), a consciência fonológica pode ser aprimorada valendo-se do desenvolvimento conjunto e inter-relacionado do aspecto cognitivo e da linguagem oral. Além disso, outros mecanismos componentes do processamento e da organização da linguagem atuam de forma subjacente ao desenvolvimento da consciência fonológica, como a memória fonológica e o acesso fonológico ao léxico mental. Ainda, outros fatores, como a idade, a capacidade cognitiva e o nível de escolaridade interferem em tarefas de consciência fonológica.

A consciência fonológica parte da identificação de palavras, rimas, sílabas, para elementos mais discretos, os fonemas. Dessa forma, a criança em idade pré-escolar tende a apresentar dificuldades de segmentar palavras em unidades pequenas, principalmente quando a unidade considerada for um fonema. As unidades maiores, como a sílaba e a rima, são mais facilmente manipuladas por ela.

\section{Metodologia}

Inicialmente, esta pesquisa foi aprovada pelo Comitê de Ética em Pesquisa do Centro de Ciências da Saúde da Universidade Federal de Santa Maria (UFSM), com cadastro sob o número 073/03.

\subsection{Sujeitos e coleta de dados}

Realizou-se a seleção da amostra e a coleta dos dados em dois locais: no Centro de Estudos da Linguagem e Fala (CELF), vinculado ao Serviço de Atendimento Fonoaudiológico (SAF), do Curso de Fonoaudiologia da UFSM; e em duas escolas públicas do município de Santa Maria (RS).

A amostra deste estudo foi formada por 28 crianças com diagnóstico de desvio fonológico. Destas, 14 aguardavam atendimento fonoaudiológico no CELF, e 14 foram selecionadas em escolas públicas. Ao total: 8 (29\%) eram do sexo feminino; 20 (71\%) do 
sexo masculino (idade entre 4:0 e 6:7 anos). Todas as crianças participantes da pesquisa não estavam alfabetizadas e apresentavam hipótese de escrita pré-silábica.

A amostra foi dividida em dois grupos pela classificação de severidade do desvio fonológico (KESKE-SOARES, 2001). Um grupo formado por 21 crianças (idade média de 5:4), que apresentava desvio fonológico com características iniciais e atrasadas (DFIA) desvio mais severo-, e outro grupo constituído de 7 crianças (idade média de 5:9), com desvio fonológico com características atrasadas (DFA)-desvio mais leve.

A caracterização da amostra segundo faixa etária, sexo e classificação da tipologia do desvio fonológico está representada no Quadro 1.

Quadro 1: Caracterização da amostra segundo faixa etária, sexo e classificação do desvio fonológico em
desvio fonológico com características iniciais e atrasadas (DFIA) e desvio fonológico com características
atrasadas (DFA) (n=28)
\begin{tabular}{|ccccccc|}
\hline Sexo & \multicolumn{4}{c}{ Classificação do desvio fonológico } \\
Faixa etária & Feminino & Masculino & Total & DFIA & DFA & Total \\
4 anos & 1 & 4 & 5 & 5 & 0 & 5 \\
5 anos & 7 & 7 & 14 & 10 & 4 & 14 \\
6 anos & 0 & 9 & 9 & 6 & 3 & 9 \\
Total & 8 & 20 & 28 & 21 & 7 & 28 \\
\hline
\end{tabular}

Foram estabelecidos os seguintes critérios para a inclusão das crianças na amostra: estarem autorizadas pelos pais ou responsáveis a participar da pesquisa por meio do Termo de Consentimento Livre e Esclarecido; apresentarem diagnóstico de desvio fonológico; não terem sido submetidas a tratamento fonoaudiológico anterior; não estarem alfabetizadas; terem entre 4 anos e 6 anos e 11 meses de idade; não apresentarem alterações evidentes nos aspectos neurológico, cognitivo, psicológico e/ou emocional; não apresentarem alterações evidentes na triagem fonoaudiológica quanto à linguagem (exceto no componente fonológico e no sistema estomatognático) e não apresentarem alterações na triagem audiológica.

Algumas crianças foram encaminhadas ao CELF, pelo setor de triagem fonoaudiológica do SAF, entre 2003 e maio de 2004- período em que aguardavam vaga na lista de espera para atendimento fonoaudiológico.

Todas as crianças do CELF foram avaliadas no mesmo ambiente (em uma sala silenciosa). Na primeira sessão, realizou-se a exposição do Termo de Consentimento Livre e Esclarecido aos pais ou responsáveis, ao passo que as crianças autorizadas foram submetidas a interação e triagem fonoaudiológica. Na segunda sessão, as crianças, que 
passaram na triagem fonoaudiológica, foram avaliadas quanto à análise fonológica, à memória de trabalho e iniciada a avaliação de consciência fonológica. Na terceira e, se necessária, quarta sessão, foi concluída a avaliação da consciência fonológica.

Todas as crianças selecionadas foram encaminhadas e compareceram para avaliação otorrinolaringológica e audiológica no SAF, as quais fazem parte da rotina de avaliações complementares para crianças em atendimento no CELF.

Considerando o número de crianças (em conformidade com os critérios de inclusão da amostra) com desvio fonológico que ingressaram no CELF (14), no período inicial da coleta, foi necessário selecionar outras crianças em duas escolas: uma municipal; outra estadual - do município de Santa Maria (RS). A escolha por escolas públicas visava igualar o nível sócio-econômico-cultural das crianças, uma vez que a população que procura o SAF não estuda em escolas particulares.

Nas escolas, todas as crianças autorizadas (14) foram avaliadas em ambientes silenciosos: sala de Orientação Educacional e de Vídeo. 0 mesmo procedimento do SAF, quanto às sessões, foi aplicado nas Escolas: interação, triagem fonoaudiológica, avaliação fonológica (da memória de trabalho e da consciência fonológica). As crianças compareceram para avaliação audiológica no SAF.

Os pais ou responsáveis foram informados sobre o atendimento oferecido pelo SAF. Foram fornecidos esclarecimentos às professoras a respeito dos encaminhamentos.

\subsection{Avaliações}

Inicialmente, realizou-se triagem fonoaudiológica com objetivo de confirmar diagnóstico de desvio fonológico, observando-se o desenvolvimento da linguagem, o sistema estomatognático, a articulação da fala e os aspectos neurológico, cognitivo, psicológico e/ou emocional.

A avaliação audiológica realizou-se no Setor de Audiologia Infantil do SAF, com o intuito de averiguar se os níveis de audição das crianças estavam dentro dos padrões de normalidade de modo que não interferissem na fala.

Para a avaliação fonológica, foi coletada amostra da fala através da nomeação e fala espontânea, realizada com a utilização do instrumento Avaliação Fonológica da Criança (AFC)- proposto por Yavas, Hernandorena e Lamprecht (1991)- possibilitando 
a eliciação de todos os fones contrastivos do português, em todas as posições que podem ocorrer em relação à estrutura da sílaba e da palavra. Além disso, com propósito de classificar o desvio fonológico de acordo com o grau de severidade, adotou-se a classificação qualitativa proposta por Keske-Soares (2001).

Para avaliação da memória de trabalho, levaram-se em conta dois de seus componentes: o executivo central e o circuito fonológico. Para avaliar o circuito fonológico, aplicou-se tarefa de repetição de não palavras (KESSLER, 1997), e, para avaliação do funcionamento do executivo central, com o circuito fonológico, empreendeu-se tarefa de repetição de sequências de dígitos - subteste 5 de memória sequencial auditiva do Teste Illinois de Habilidades Psicolinguísticas (ITPA)-, adaptação e padronização brasileira realizada por Bogossian e Santos (1977).

Para avaliação da consciência fonológica, utilizou-se do teste Consciência Fonológica: Instrumento de Avaliação Sequencial - CONFIAS (MOOJEN et al., 2003), o qual é indicado para crianças não alfabetizadas e avalia a consciência fonológica de forma abrangente, por meio de variadas tarefas silábicas e fonêmicas que garantem, de modo sequencial, acesso aos diferentes níveis de consciência fonológica, com escala crescente de complexidade das tarefas, partindo de tarefas simples a mais complexas.

Esse instrumento de avaliação considera as características do português brasileiro e possibilita a investigação das capacidades fonológicas relacionando-as com as hipóteses de escrita- formuladas por Ferreiro e Teberosky (1991 apud MOOJEN et al., 2003)- agrupadas em quatro níveis gerais: pré-silábico, silábico, silábico-alfabético e alfabético.

\subsection{Análise dos dados}

Realizou-se análise estatística de correlação dos resultados obtidos das avaliações de memória de trabalho e de consciência fonológica na amostra total, através do teste paramétrico Coeficiente de Correlação de Pearson e do teste não paramétrico Coeficiente de Correlação de Spearman. Para verificar se as diferenças de médias entre os grupos DFIA e DFA eram significativas, utilizaram-se do teste $\mathrm{t}$ paramétrico e o teste não paramétrico de Kruskal-Wallis, quando, pelo menos, uma das medidas não seguia uma distribuição normal. Todos os testes com valores significativos para $\mathrm{p}<0,05$. 
Em razão do fato de que, neste trabalho, não foi formado um grupo controle, buscou-se na literatura estudos sobre memória de trabalho realizados com crianças com desenvolvimento fonológico normal, considerando-se a faixa etária, a escolaridade e a metodologia de avaliação semelhantes (KESSLER, 1997; BRODACZ, 1998; JERONYMO; GALERA, 2000; LINASSI, 2001, 2002; LINASSI et al., 2004). Da mesma forma, para a consciência fonológica, os resultados deste estudo foram comparados com os valores de desempenho de crianças com desenvolvimento fonológico normal, propostos no teste CONFIAS (MOOJEN et al., 2003). O estudo de Cardoso, Silva e Pereira (2013) relaciona memória de trabalho e consciência fonológica em sujeitos com desenvolvimento normal.

Os resultados obtidos também foram comparados com estudos brasileiros que investigaram crianças com desvio fonológico, com habilidades em consciência fonológica (VIEIRA, 2001; MORALES; MOTA; KESKE-SOARES, 2002; DIAS; MOTA; MEZZOMO, 2009; KAMINSK; MOTA; CIELO, 2011; STEFANINI et al., 2013) e com memória de trabalho (RAMOS, 1996; BRODACZ, 1998; JERONYMO; GALERA, 2000; LINASSI, 2002; LINASSI et al., 2004, 2005).

\section{Resultados e discussão}

\subsection{Resultados do desempenho da amostra total nas medidas de memória de trabalho e de consciência fonológica}

Na Tab. 1, apresentaram-se os resultados da amostra total referentes às médias, ao desvio padrão, aos valores mínimo e máximo das medidas analisadas.

Tabela 1 - Estatística descritiva da idade e das habilidades apresentadas pelas crianças da amostra total nas tarefas de memória de trabalho e de consciência fonológica (n=28)

\begin{tabular}{ccccc}
\hline Medidas & Média & Desvio Padrão & Mínimo & Máximo \\
\hline Idade $^{1}$ & 66,86 & 8,28 & 48 & 79 \\
Não-palavras $^{2}$ & 2,11 & 0,88 & 1 & 4 \\
Dígitos $^{3}$ & 3,39 & 0,83 & 2 & 5 \\
CFTotal $^{4}$ & 17,57 & 5,80 & 7 & 28 \\
CSílabas $^{5}$ & 14 & 4,63 & 7 & 24 \\
CFonemas $^{6}$ & 3,57 & 2,74 & 0 & 9 \\
\hline
\end{tabular}

Legenda: 1. Idade em meses; 2 . Tarefa de repetição de não palavras (n. ${ }^{\circ}$ de sílabas repetidas corretamente, escore de 1 a 6 sílabas); 3. Tarefa de repetição de sequências de dígitos (n.o de dígitos repetidos na sequência, escore de 2 a 7 dígitos); 4. Total de acertos em consciência fonológica (escore máximo = 70); 5 . Acertos em consciência de sílabas (escore máximo $=40$ ); 6 . Acertos em consciência de fonemas (escore máximo $=30$ ). 
Quanto à memória de trabalho (com base na Tab. 1), ao confrontar os resultados obtidos referentes ao desempenho das crianças com desvio fonológico em memória fonológica (repetição de não palavras) com outras pesquisas (KESSLER, 1997; BRODACZ, 1998; JERONYMO; GALERA, 2000; LINASSI, 2001, 2002), verificou-se que a amostra teve desempenho inferior na memória fonológica quando comparada às crianças com desenvolvimento fonológico normal.

Ainda, de acordo com a Tab. 1, ao confrontar resultados de estudos brasileiros (KESSLER, 1997; JERONYMO; GALERA, 2000; LINASSI, 2001, 2002; LINASSI et al., 2005), concernentes à tarefa de repetição de dígitos, com os resultados da amostra desta pesquisa (em que a média foi de três dígitos repetidos), verificou-se que as crianças com desvio fonológico também tiveram evidente desempenho inferior na memória de trabalho em relação às crianças com desenvolvimento normal nas pesquisas de Linassi (2001, 2002) e Linassi et al. (2004). Entretanto, as crianças com desvio fonológico tiveram desempenho mais próximo ao das crianças com desenvolvimento normal, segundo as pesquisas de Kessler (1997), Jeronymo e Galera (2000).

Observou-se, portanto, que, para o desempenho de crianças com desenvolvimento normal na tarefa de repetição de dígitos no português brasileiro, foram encontrados valores de até quatro dígitos para crianças de 3 anos (LINASSI, 2001), e valores entre três e seis dígitos para crianças entre 4 e 7 anos (KESSLER, 1997; JERONYMO; GALERA, 2000; LINASSI, 2002; LINASSI et al., 2004).

Tendo em vista a dificuldade em verificar se o desempenho em repetição de dígitos está adequado ou não conforme as pesquisas com crianças normais, o escore escalar do teste ITPA mostrou-se uma medida importante, pois através dele pôde-se classificar, nesta tarefa, o desempenho das crianças da amostra: em não esperado ou esperado (de acordo com a idade cronológica e os escores esperados para cada faixa etária). Por exemplo, para uma criança que conseguiu repetir o máximo de três dígitos, não se pode dizer como foi seu desempenho na tarefa de repetição de dígitos em relação ao desempenho das crianças normais. Pode-se verificar se o escore escalar obtido está adequado ou não em comparação com crianças da mesma faixa etária, uma vez que o escore escalar é uma transformação linear da pontuação máxima obtida em todas as 
sequências de dígitos apresentadas (escore bruto) - não serve apenas para indicar o número máximo de dígitos obtido.

As crianças com desvio fonológico da amostra demonstraram pior desempenho em escore escalar quando comparadas às crianças com desenvolvimento fonológico normal das pesquisas de Kessler (1997) e Linassi et al. (2004). Esse resultado foi semelhante ao das pesquisas de Ramos (1996) e Linassi et al. (2004), com crianças com desvio fonológico que também apresentaram desempenho não esperado no escore escalar do teste ITPA.

Quanto ao desempenho das crianças com desvio fonológico nas habilidades em consciência fonológica, verificou-se que este foi inferior quando comparado ao de crianças com o sistema fonológico esperado da pesquisa de Moojen et al. (2003). Esse pior desempenho em consciência fonológica das crianças com desvio fonológico desta amostra concorda com os resultados de outras pesquisas internacionais e nacionais (WEBSTER; PLANTE, 1992; BIRD; BISHOP; FREEMAN, 1995; LOWE; WEITZ, 1996; WEBSTER et al., 1997; VIEIRA, 2001; MORALES; MOTA; KESKE-SOARES, 2002; KAMINSKI; MOTA; CIELO, 2011; STEFANINI et al., 2013).

Webster e Plante (1992) relataram que o desvio fonológico pode afetar a habilidade da criança em manter informações fonológicas na memória de trabalho para realizar tarefas de consciência fonológica. Bird, Bishop e Freeman (1995) mencionaram que crianças com desvio fonológico apresentam dificuldade no que se refere à caracterização dos segmentos da fala: não percebem que uma sílaba pode ser analisada em unidades fonológicas menores. Para Lowe e Weitz (1996), algumas crianças com distúrbios fonológicos têm déficts no conhecimento do seu sistema fonológico.

Webster et al. (1997), Vieira (2001), Morales, Mota e Keske-Soares (2002) constataram que crianças com desvio fonológico tiveram desempenho significativamente pior que as crianças sem alteração de fala em tarefas de consciência fonológica. Kaminski, Mota e Cielo (2011) e Stefanini et al. (2013) verificaram que a maioria das crianças com desvio fonológico apresentou desempenho inferior ao esperado para sua faixa etária na avaliação da consciência fonológica, bem como quando comparadas aos participantes com aquisição típica de linguagem.

0 pior desempenho em consciência fonológica das crianças com desvio fonológico desta amostra, com média de idade de 5:6, discordou do resultado 
encontrado por Oliveira et al. (2004), de quatro crianças (idade entre 6:8 e 9:3), com desvio fonológico, apenas uma teve desempenho inferior em consciência fonológica para seu nível de escrita. Observou-se que essas crianças eram mais velhas que as desta amostra, ao passo que a comparação dos resultados foi realizada avaliando o desempenho individual - e não o grupo.

Magnusson (1990) referiu que geralmente as comparações entre crianças com desvios e crianças sem desvios de linguagem em nível de grupo mostram que as crianças com desvios têm um nível de consciência metalinguística inferior ao das crianças normais. Mesmo que a maioria das crianças com desvios linguísticos tenha menos consciência que crianças sem desvios, quando submetidas a uma análise individual, há, entre as com desvios, as que apresentam uma consciência igual ou até superior à de crianças normais.

Quanto aos resultados de consciência fonológica, observou-se, ainda, de modo geral, que as tarefas mais acessíveis para as crianças da amostra foram as que envolveram consciência silábica e identificação de rima, enquanto as tarefas de consciência fonêmica foram as mais difíceis.

Constatou-se, ainda, que todas as tarefas de consciência silábica precederam as de consciência fonêmica, levando-se em conta as tarefas silábicas e fonêmicas separadamente: desde a síntese, passando pela segmentação, identificação, produção, exclusão, até chegar à transposição.

Os dados da Tab. 2, referentes à amostra total, mostraram os valores de correlação entre as medidas de idade, a memória de trabalho e a consciência fonológica.

Tabela 2 - Correlação entre idade com acertos obtidos das tarefas de memória de trabalho e de consciência fonológica na amostra total $(n=28)$

\begin{tabular}{|c|c|c|c|c|c|c|c|}
\hline & Idade & NP & DIG & EE & CFTotal & CSílabas & CFonemas \\
\hline Idade & & & & & & & \\
\hline NP & $0,47 B^{*}$ & - & & & & & \\
\hline DIG & $0,16 \mathrm{~B}$ & $0,32 C^{*}$ & & 1 & & & \\
\hline EE & $0,04 \mathrm{~B}$ & $0,29 B$ & $0,87 \mathrm{~B}^{*}$ & & 1 & & \\
\hline CFTotal & $0,27 \mathrm{~A}$ & $0,18 \mathrm{~B}$ & $0,14 \mathrm{~B}$ & $0,22 \mathrm{~B}$ & 1 & & \\
\hline CSílabas & $0,27 \mathrm{~A}$ & $0,42 B^{*}$ & $-0,01 B$ & $0,08 \mathrm{~B}$ & $0,89 A^{*}$ & 1 & \\
\hline CFonemas & $0,10 \mathrm{~A}$ & $-0,31 B$ & $0,17 \mathrm{~B}$ & $0,22 \mathrm{~B}$ & $0,62 A^{*}$ & $0,19 \mathrm{~A}$ & 1 \\
\hline
\end{tabular}


Na Tab. 2, observando-se as medidas de idade e memória de trabalho, pôde-se verificar correlação estatisticamente significativa $(\mathrm{p}<0,05)$ entre a idade e a tarefa de repetição de não palavras. Houve, ainda, correlação estatisticamente significativa $(\mathrm{p}<0,05)$ entre as tarefas de repetição de não palavras e repetição de dígitos, e entre a repetição de dígitos e o escore escalar.

0 resultado obtido neste estudo, de que a idade e a tarefa de repetição de não palavras estão correlacionadas significativamente, está de acordo com o referido por Gathercole e Baddeley (1993): a memória fonológica, mais especificamente o efeito de extensão da palavra, aumenta em consequência da idade. E por Gathercole et al. (1994), ao referir que o número de acertos nas tarefas de memória fonológica aumenta em consequência da idade. Esse resultado concorda com o de Cielo (2001), ao constatar que o desempenho das crianças ao realizar tarefas de consciência fonológica, que envolvia processamento de maior número de elementos, melhorou em virtude da idade.

Este estudo indicou que a idade não foi correlacionada de forma significativa com a tarefa de repetição de dígitos, mostrando que não foi constatado aumento evidente na capacidade da memória de trabalho entre as idades de 4 e 6 anos, quando considerado o aumento na extensão de dígitos repetidos corretamente.

Conforme os valores apresentados entre as medidas de idade e consciência fonológica, na Tab. 2, verificou-se que a idade não foi correlacionada significativamente com as habilidades em consciência fonológica. Observou-se que existe correlação estatisticamente significativa $(\mathrm{p}<0,05)$ entre as medidas de consciência fonológica total e a consciência de sílabas, e entre a consciência fonológica total e a consciência de fonemas.

0 resultado encontrado, na Tab. 2 , de que a idade não está correlacionada significativamente com a consciência fonológica, não condiz com pesquisas de autores como Carvalho, Alvarez e Caetano (1998): o desenvolvimento da consciência fonológica está relacionado somente com a idade cronológica. Estes propuseram resultados em consciência fonológica, para crianças de 5 a 10 anos, com desempenhos que aumentaram em razão da idade delas. E Vieira et al. (2004) ao referir correlação entre a consciência fonológica e o aumento da idade em crianças de 4 a 7 anos com desvio fonológico. No entanto, converge com Cielo (2001), que verificou que nas faixas etárias entre 4 e 6 anos não houve crescimento muito diferenciado da consciência fonológica, 
mas, sim, entre estas idades em comparação com as idades de 7 e 8 anos, quando sob influência da alfabetização no desenvolvimento da consciência fonológica, principalmente da consciência fonêmica.

Esta pesquisa investigou a consciência fonológica de crianças de diferentes faixas etárias (4, 5 e 6 anos), mas que tinham o mesmo nível de hipótese de escrita: a hipótese pré-silábica. Fato que deve ter colaborado para o resultado encontrado: o aumento da idade não foi significativo para o desempenho nas tarefas de consciência fonológica.

Ainda na Tab. 2, pode-se verificar a correlação significativa entre a tarefa de repetição de não palavras e a tarefa de consciência de sílabas, evidenciando que as habilidades em memória fonológica e em consciência fonológica estão correlacionadas de maneira significativa em crianças com desvio fonológico.

Esse resultado corrobora o resultado encontrado por vários autores, como Mann e Liberman (1984), que sugeriram existir correlação moderada entre consciência fonológica e memória fonológica. Couture e McCauley (2000) mencionaram que a consciência fonológica está relacionada à memória fonológica em crianças com desvio fonológico, sendo provável que ela tenha papel importante nas tarefas que solicitem a consciência fonológica. Para resolver as tarefas de consciência fonológica, é necessário que o material verbal seja mantido na memória de curto prazo. Capovilla, Gutschow e Capovilla (2002) apontaram uma correlação positiva entre consciência fonológica e memória de trabalho, mas as habilidades em memória fonológica não pareceram ser função do treino da consciência fonológica. Para Oakhill e Kyle (2000), a memória de trabalho prediz o desempenho em consciência fonológica na tarefa de categorização do som, a qual envolve rima, mas não prediz na tarefa de exclusão de fonema. Isso significa que as tarefas de consciência fonológica exigem, para sua análise, diferentes níveis de memória de trabalho ou de memória de curto prazo, havendo, assim, uma inter-relação entre memória de trabalho e algumas tarefas de consciência fonológica. Cardoso, Silva e Pereira (2013) encontraram uma correlação significativa entre as habilidades em consciência fonológica e memória de trabalho para sujeitos com desenvolvimento normal.

A correlação significativa entre memória fonológica e consciência fonológica encontrada nesta pesquisa diverge da de Capovilla e Capovilla $(1997,2000)$, que não encontraram evidências corroborativas da hipótese de uma relação entre a consciência 
fonológica e a memória fonológica, dado que o treino de consciência fonológica não produziu efeitos sobre a memória fonológica.

\subsection{Resultados do desempenho dos grupos DFIA e DFA nas medidas de memória de trabalho e de consciência fonológica}

Na Tab. 3 apresentam-se os resultados do desempenho dos grupos DFIA e DFA referentes às médias, ao desvio padrão e aos valores mínimo e máximo das medidas, bem como das significâncias das diferenças de médias.

Tabela 3 - Estatística descritiva e significâncias das diferenças de médias da idade e dos resultados dos desempenhos apresentados pelas crianças dos grupos DFIA (n=21) e DFA (n=7) nas tarefas de memória de trabalho (não palavras e dígitos) e de consciência fonológica (total, sílabas e fonemas)

\begin{tabular}{cccccc}
\hline \multirow{2}{*}{ Medidas } & \multicolumn{2}{c}{ DFIA } & \multicolumn{2}{c}{ DFA } & Diferença \\
& Média & DP & Média & DP & \\
\hline Idade & 65,43 & 8,76 & 71,14 & 4,91 & $\mathrm{p}=0,1152 \mathrm{D}$ \\
Não-palavras & 1,95 & 0,86 & 2,57 & 0,79 & $\mathrm{p}=0,1000 \mathrm{E}$ \\
Dígitos & 3,33 & 0,86 & 3,57 & 0,79 & $\mathrm{p}=0,4313 \mathrm{E}$ \\
CFTotal & 16,05 & 5,79 & 22,14 & 2,67 & $\mathrm{p}=0,0130 \mathrm{D}^{*}$ \\
CSílabas & 13,14 & 4,93 & 16,57 & 2,23 & $\mathrm{p}=0,0784 \mathrm{E}$ \\
CFonemas & 2,90 & 2,66 & 5,57 & 1,99 & $\mathrm{p}=0,0197 \mathrm{E}^{*}$ \\
\hline
\end{tabular}

Legenda: *Valores de correlação significativos para $\mathrm{p}<0,05$.

Na Tab. 3, mostra-se a estatística descritiva com os resultados dos grupos "desvio fonológico mais grave" (DFIA) e "desvio fonológico mais leve" (DFA). Nas tarefas para avaliar a habilidade em memória de trabalho, a média de sílabas repetidas corretamente na repetição de não palavras foi de 2 sílabas para o grupo DFIA e de 3 para o grupo DFA; a média do número de dígitos repetidos corretamente foi de 3 dígitos para o grupo DFIA e de 4 dígitos para o grupo DFA. Nas tarefas para avaliar a habilidade em consciência fonológica, a média de acertos totais obtida foi de 16 para o grupo DFIA e de 22 para o grupo DFA; a média na consciência de sílabas foi de 13 acertos para o grupo DFIA e de 17 para o grupo DFA, enquanto na consciência de fonemas a média foi de 3 acertos para o grupo com desvio mais severo e de 6 acertos para o grupo com desvio mais leve. 
Na Tab. 3, quanto à severidade do desvio fonológico e as habilidades em memória de trabalho, observou-se que a média na repetição de não palavras e na repetição dos dígitos do grupo DFIA foi inferior à do grupo DFA, mas sem diferença significativa.

Esse resultado corrobora os resultados de Linassi (2002) e Linassi et al. (2005), quanto à relação entre o grau de severidade do desvio fonológico e a habilidade em memória de trabalho, avaliada com a prova de repetição de dígitos. No entanto, há discordância quanto ao grau de severidade do desvio fonológico estar relacionado com a memória fonológica.

Nas tarefas de consciência fonológica, média de acertos totais, na consciência de sílabas e na consciência de fonemas das crianças do grupo DFIA foi inferior quando comparado ao grupo DFA, com diferença estatisticamente significativa na consciência fonológica total e na consciência fonêmica.

Os resultados desta pesquisa estão de acordo com os de Webster e Plante (1992), que indicaram uma íntima relação entre as habilidades em consciência fonológica e a produção fonológica, com um estudo envolvendo crianças com desvio fonológico e sem alteração de fala, no qual observaram que a severidade do desvio fonológico, medido pelo PCC, pode ser um fator preditor importante do desempenho nas tarefas de consciência fonológica. Segundo Bird, Bishop e Freeman (1995), a severidade e a persistência do desvio fonológico afetam significativamente a consciência fonológica e a alfabetização das crianças com alteração de fala, visto que a severidade do desvio é fator preditivo importante para a alfabetização. Dias, Mota e Mezzomo (2009) identificaram uma possível relação entre o desempenho nas tarefas de consciência fonológica e as diferentes gravidades do desvio fonológico, principalmente quando classificados de forma qualitativa.

0 resultado desta pesquisa mostrou haver correlação entre a severidade do desvio fonológico e a consciência fonológica, principalmente nos escores totais, e na consciência fonêmica discorda do proposto por Magnusson (1990), que referiu não ser possível prever a consciência fonológica com base no grau de desvio apresentado na produção da fala.

Ao estudar a relação entre consciência fonológica, avaliada com a Prova de Consciência Fonológica (CAPOVILLA; CAPOVILLA, 1997), e grau de severidade da fala, avaliado pelo PCC, Vieira et al. (2004) chegaram a resultados discordantes ao obtido 
nesta pesquisa, pois verificou-se que não houve correlação significativa entre o grau de severidade do desvio fonológico com a consciência fonológica. Não obstante, constatouse que o desempenho de crianças com desvio fonológico em tarefas de consciência fonológica não está diretamente associado ao grau de severidade do desvio fonológico.

Uma possível explicação para os resultados discordantes obtidos na correlação entre consciência fonológica e a severidade do desvio fonológico, apresentados no estudo de Vieira et al. (2004) e de Dias, Mota e Mezzomo (2009), pode ser devido às diferentes metodologias utilizadas, dado que as avaliações, tanto da classificação da severidade do desvio fonológico quanto da consciência fonológica, eram diferentes.

Pôde-se comprovar, portanto, que a severidade do desvio da fala está relacionada positivamente com o desempenho das crianças com desvio fonológico nas habilidades em consciência fonológica, posto que o grupo DFIA apresentou um desempenho inferior ao grupo DFA, com diferença significativa. Entretanto, a severidade da alteração de fala não influenciou de maneira significativa o desempenho das crianças com desvio fonológico em tarefas que avaliam a memória de trabalho.

Neste estudo, percebeu-se que a maior severidade da fala não implicou, necessariamente, pior memória de trabalho, mas interferiu significativamente na consciência fonológica. Crianças com DFIA podem ter dificuldade em consciência fonológica, mais do que crianças do grupo DFA - quanto maior a desorganização do sistema fonológico, pior para a criança refletir sobre a linguagem. Em contrapartida, as dificuldades em memória de trabalho não foram mais evidentes em crianças com desvio fonológico mais severo do que nas com desvio fonológico mais leve.

\section{Conclusões}

Com base nos resultados apresentados e discutidos, podem-se estabelecer as seguintes conclusões:

a) o desempenho das crianças com desvio fonológico, especificamente em memória fonológica - componente da memória de trabalho avaliada pela tarefa de repetição de não palavras -, foi inferior quando comparado ao de crianças com desenvolvimento fonológico normal; 
b) o desempenho das crianças com desvio fonológico em memória de trabalho, que inclui o executivo central e a memória fonológica, avaliada pela tarefa de repetição de dígitos, foi inferior ao encontrado em crianças com desenvolvimento fonológico normal em algumas pesquisas e próximo em outras;

c) na avaliação de consciência fonológica, as crianças com desvio fonológico tiveram desempenho inferior quando comparado ao de crianças sem alterações fonológicas, mas seu desenvolvimento segue diferentes níveis de complexidade, de modo semelhante ao de crianças normais, aparecendo primeiro a consciência silábica, depois a rima e por último a consciência fonêmica;

d) houve correlação significativa entre a tarefa de repetição de não palavras e a tarefa de consciência de sílabas, evidenciando que as habilidades em memória fonológica e em consciência fonológica estão correlacionadas de forma significativa nas crianças com desvio fonológico;

e) houve correlação significativa entre a idade e a memória fonológica;

f) verificou-se que a severidade do desvio da fala não influencia de maneira significativa o desempenho na habilidade em memória de trabalho, mas está relacionada com o desempenho das crianças com desvio fonológico nas habilidades em consciência fonológica.

Espera-se, com os resultados deste estudo ${ }^{1}$, contribuir para a pesquisa e para a atuação clínica fonoaudiológica, no sentido de incluir avaliações e estimulação das habilidades em processamento fonológico à terapia fonológica de crianças com desvio fonológico. E, ainda, despertar o interesse em estudos na área da linguística, especialmente na fonologia e na psicolinguística.

\section{Referências}

ACOSTA, Víctor et al. Avaliação da linguagem: teoria e prática do processo de avaliação do comportamento linguístico infantil. São Paulo: Santos, 2003.

\footnotetext{
1 Esta pesquisa foi realizada no Centro de Estudos da Linguagem e da Fala (CELF) do Curso de Fonoaudiologia da Universidade Federal de Santa Maria (RS), com orientação da Professora Dra. Helena Bolli Mota e da Professora Dra. Marcia Keske-Soares, às quais transmito meus agradecimentos pela orientação no desenvolvimento do presente trabalho.
} 
AVILA, Clara Regina. Consciência fonológica. In: FERREIRA, Léslie Piccolotto; BEFILOPES, Debora; LIMONGI, Suelly Cecília Olivan (Org.). Tratado de fonoaudiologia. São Paulo: Roca, p. 815-824, 2004.

BADDELEY, Alan David. Working memory. Oxford: ClaridonPress, 1986.

Human memory: theory and practice. Boston: Allyn and Bacon, 1998.

. Working memory and language: an overview. Journal of Communication Disorders, Philadelphia, v. 36, n. 3, p. 189-208, 2003.

BIRD, J.; BISHOP, Dorothy V. M.; FREEMAN, N. H. Phonological awareness and literacy development in children with expressive phonological impairments. Journal of speech and hearing research, Rockville, v. 38, p. 446-462, 1995.

BOGOSSIAN, Maria Alice Dias da Silva; SANTOS, Maria Judith. Adaptação brasileira do teste Illinois de habilidades psicolinguísticas. Florianópolis: Tamasa, 1977.

BRODACZ, Raquel. Um estudo sobre a memória de trabalho em crianças com desvios fonológicos. 1998. 83 f. Dissertação (Mestrado em Letras) - Pontifícia Universidade Católica do Rio Grande do Sul, Porto Alegre, 1998.

CAPELLINI, Simone Aparecida; CIASCA, Sylvia Maria. Avaliação da consciência fonológica em crianças com distúrbio específico da leitura e escrita e distúrbio de aprendizagem. Temas sobre desenvolvimento, São Paulo, v. 8, n. 48, p. 17-23, 2000.

CAPOVILLA, Alessandra; CAPOVILLA, Fernando César. Treino da consciência fonológica e seu impacto em habilidades fonológicas, de leitura e ditado de pré-3 a segunda série. Ciência cognitiva: teoria, pesquisa e aplicação, Rio de Janeiro, v. 1, n. 2, p. 461-532, 1997.

Problemas de leitura e escrita. São Paulo: Memnon, 2000.

CAPOVILLA, Alessandra; GÜTSCHOW, Cláudia Regina; CAPOVILLA, Fernando César. Identificação de perfis cognitivos preditivos de dificuldades de leitura e escrita. In: MACEDO, Elizeu Coutinho et al. Tecnologia em (re)habilitação cognitiva 2002: um novo olhar para avaliação e intervenção. São Paulo: Centro Universitário São Camilo, 2002. p. 173-188.

CARVALHO, Isabel Albuquerque M.; ALVAREZ, Ana Maria Maaz Acosta; CAETANO, Aparecida Liberato. Perfil das habilidades fonológicas. São Paulo: Via Lettera, 1998.

CARDOSO, Andreia; SILVA, Mônica; PEREIRA, Mônica. Consciência fonológica e a memória de trabalho de crianças com e sem dificuldades na alfabetização. CoDAS, São Paulo, v. 25, n. 2, p. 110-114, 2013.

CIELO, Carla Aparecida. Habilidades em consciência fonológica em crianças de 4 a 8 anos de idade. 2001. 144 f. Tese (Doutorado em Linguística Aplicada) -Pontifícia Universidade Católica do Rio Grande do Sul, Porto Alegre, 2001. 
COUTURE, Ashley E.; McCAULEY, Rebecca J. Phonological working memory in children with phonological impairment. Clinical linguistic \& phonetics, London, v. 14, n. 7, p. 499$517,2000$.

DIAS, Roberta; MOTA, Helena; MEZZOMO, Carolina. A consciência fonológica e a consciência do próprio desvio de fala nas diferentes gravidades do desvio fonológico. Rev. CEFAC, Rio de Janeiro, v. 11, n. 4, p. 561-570, 2009.

EYSENCK, Michael W; KEANE, Mark T. Psicologia cognitiva: um manual introdutório. Porto Alegre: Artes Médicas, 1994.

GATHERCOLE, Susan E.; BADDELEY, Alan David. Working memory and language. Hove: Lawrence Erlbaum, 1993.

GATHERCOLE, Susan et al. The children's test of nonword repetition: a test of phonological working memory. In: GATHERCOLE, Susan; McCARTHY, Rosaleen. Memory tests and techniques. Hove, UK: Lawrence Erlbaum Associates, 1994, p.103-128.

GOMBERT, Jean Émile. Metalinguistic development. Chicago: University of Chicago Press, 1992.

GRUNWELL, Pamela. The nature of phonological disability in children. London: Academic Press, 1981.

INGRAM, David. Phonological disability in children. London: Edward Arnold, 1976.

IZQUIERDO, Iván. Memória. Porto Alegre: Artmed, 2002.

JERONYMO, Rosângela; GALERA, César Alexis. A relação entre a memória fonológica e habilidade linguística de crianças de 4 a 9 anos. Pró-fonorevista de atualização científica, Barueri (SP), v. 12, n. 2, p. 55-60, 2000.

KAMINSKI, Tassiana Isabel; MOTA, Helena Bolli; CIELO, Carla Aparecida. Consciência fonológica e vocabulário expressivo em crianças com aquisição típica da linguagem e com desvio fonológico. Revista CEFAC, Rio de Janeiro, v. 13, n. 5, p. 813-824, 2011.

KESKE-SOARES, Marcia. Terapia fonoaudiológica fundamentada na hierarquia implicacional dos traços distintivos aplicada em crianças com desvios fonológicos. 2001. 193 f. Tese (Doutorado em Letras) - Pontifícia Universidade Católica do Rio Grande do Sul, Porto Alegre, 2001.

KESSLER, Themis Maria. Estudo da memória operacional em pré-escolares. 1997. 36f. Dissertação (Mestrado em Distúrbios da Comunicação Humana) - Universidade Federal de Santa Maria, Santa Maria, 1997.

LAMPRECHT, Regina Ritter et al. Aquisição fonológica do português: perfil de desenvolvimento e subsídios para terapia. Porto Alegre: Artmed, 2004. p. 167-176. 
LINASSI, Lisiane Zorzella. Avaliação da memória de trabalho em crianças de 2:11 meses a 3:11 meses. 2001. 50 f. Monografia (Especialização em Fonoaudiologia) - Universidade Federal de Santa Maria, Santa Maria, 2001.

Memória de trabalho em crianças com desvio fonológico. 2002. 87f. Dissertação (Mestrado em Distúrbios da Comunicação Humana) - Universidade Federal de Santa Maria, Santa Maria, 2002.

LINASSI, Lisiane Zorzella; KESKE-SOARES, Marcia; MOTA, Helena Bolli. Memória de trabalho em crianças com desvio fonológico. Pró-fonorevista de atualização científica, Barueri (SP), v. 16, n. 1, p. 75-82, 2004.

Habilidades de memória de trabalho e o grau de severidade do desvio fonológico. Pró-fonorevista de atualização científica, Barueri (SP), v.17, n.3, p.383-392, 2005.

LOWE, Robert J.; WEITZ, Julia Mount. Intervenção. In: LOWE, Robert J. Fonologia: avaliação e intervenção: aplicações na patologia da fala. Porto Alegre: Artes Médicas, 1996. p. 159-188.

MAGNUSSON, Eva. Consciência metalinguística em crianças com desvios fonológicos. In: YAVAS, M. (Org.) Desvios fonológicos em crianças: teoria, pesquisa e tratamento. Porto Alegre: Mercado Aberto, 1990. p. 109-148.

MANN, Virginia A.; LIBERMAN, Isabelle Y. Phonological awareness and verbal shortterm memory. Journal of learning disabilities, Austin, v. 17, n. 10, p. 592-599, 1984.

MO0JEN, Sônia et al. Consciência fonológica: Instrumento de avaliação sequencial. São Paulo: Casa do Psicólogo, 2003.

MORALES, Michele Vieira; MOTA, Helena Bolli; KESKE-SOARES, Marcia. Consciência fonológica: desempenho de crianças com e sem desvios fonológicos evolutivos. Prófonorevista de atualização científica, v.14, n.2, p. 153-164, 2002.

MOTA, Helena Bolli et al. Classificação quantitativa versus qualitativa do grau de severidade dos desvios fonológicos. In: CONGRESSO BRASILEIRO DE FONOAUDIOLOGIA, 16, 2008, Campos do Jordão. Anais... Campos do Jordão: SBFa, 2008, p. 561.

OAKHILL, Jane; KYLE, Fiona. The relation between phonological awareness and working memory. Journal of Experimental Child Psychology, New York, v. 75, p. 152-164, 2000.

OLIVEIRA, Carolina Cardoso et al. Cronologia da aquisição dos segmentos e das estruturas silábicas. In: LAMPRECHT, Regina Ritter et al. Aquisição fonológica do português: perfil de desenvolvimento e subsídios para terapia. Porto Alegre: Artmed, 2004. p. 167-176.

OLIVEIRA, Ana Gabriela et al. Nível de consciência fonológica de sujeitos com desvios fonológicos: comportamento na testagem e relação com o estado do sistema fonológico. 
In: CONGRESSO BRASILEIRO DE FONOAUDIOLOGIA, 12 E CONGRESSO SUL BRASILEIRO DE FONOAUDIOLOGIA, v. 2, 2004, Foz do Iguaçu. Anais... Foz do Iguaçu: SBFa, 2004. 1 CD-ROM.

RAMOS, Ana Paula Fadanelli. Processos de estrutura silábica em crianças com desvios fonológicos: uma abordagem não-linear. 1996. 168f. Tese (Doutorado em Letras: Área de Concentração - Linguística Aplicada) - Pontifícia Universidade Católica do Rio Grande do Sul, Porto Alegre, 1996.

SHRIBERG, Lawrence D.; KWIATKOWSKI, Joan. Phonological disorders I: a diagnostic classification system. Journal of Speech and Hearing Disorders, v. 47, p. 226-241, 1982.

SHRIBERG, Lawrence D. et al. The percentage of consonants corrects (PCC) metric: extensions and reliability data. Journal of Speech, Language, and Hearing Research. Rockville, v. 40, p. 708-722, 1997.

STACKHOUSE, Joy. Phonological awareness: connecting speech and literacy problems. In: HODSON, Barbara Williams; EDWARDS, Mary Louise. Perspectives in applied phonology. Gaithersburg Maryland: An Aspen Publication, 1997, p.157-196.

STEFANINI, Marcela et al. Desempenho em consciência fonológica por crianças com transtorno fonológico: comparação de dois instrumentos. Revista CEFAC, São Paulo, v. 15, n. 5, p. 1227-1235, 2013.

STERNBERG, Robert J. Psicologia cognitiva. Porto Alegre: Artmed, 2000.

TORGENSEN, Joseph K.; WAGNER, Richard K.; RASHOTTE, Carol A. Longitudinal studies of phonological processing and reading. Journal of learning disabilities, Austin, v. 27, n. 5, p. 276-286, 1994.

TUNMER, William E.; HARRIMAN, Michael L.; NESDALE, Andrew R. Metalinguistic abilities and beginning reading. Reading research quarterly, v. 23, n. 2, p. 134-158, 1988.

VIEIRA, Michele Gindri. Habilidades em consciência fonológica: desempenho de crianças com e sem desvios fonológicos evolutivos. 2001. 90f. Monografia (Especialização em Fonoaudiologia) - Universidade Federal de Santa Maria, Santa Maria, 2001.

VIEIRA, Michele Gindri; MOTA, Helena Bolli; KESKE-SOARES, Marcia. Relação entre idade, grau de severidade do desvio fonológico e consciência fonológica. Revista da sociedade brasileira de fonoaudiologia, São Paulo, v. 9, n. 3, p. 144-150, 2004.

WEBSTER, Penelope E.; PLANTE, Amy Solomon; COUVILlION, L. Michael. Phonologic impairment and prereading: update on a longitudinal study. Journal of Learning Disabilities, Austin, v. 30, n. 4, p. 365-375, 1997.

WEBSTER, Penelope E.; PLANTE, Amy Solomon. Effects of phonological impairment on word, syllable, and phoneme segmentation and reading. Language, speech, and hearing services in schools, Washington, v. 23, p. 176-182, 1992. 
WERTZNER, Haydée. Fonologia: desenvolvimento e alterações. In: FERNANDES, Fernanda; MENDES, Beatriz; NAVAS, Ana Luiza (Org.). Tratado de fonoaudiologia. 2. ed. São Paulo: Roca, 2009. p. 281-290.

YAVAS, Mehmet; HERNANDORENA, Carmen Lúcia; LAMPRECHT, Regina Ritter. Avaliação fonológica da criança. Porto Alegre: Artes Médicas, 1991.

Recebido em junho de 2014.

Aceito em outubro de 2014. 